\title{
Successful Early Rituximab Treatment in a Case of Systemic Lupus Erythematosus with Potentially Fatal Diffuse Alveolar Hemorrhage
}

\author{
Ju Ock Na ${ }^{a}$ Sung Hae Chang ${ }^{b}$ Ki-Hyeon Seo ${ }^{a}$ Jae Sung Choi ${ }^{a}$ Ho Sung Lee \\ Ji Won Lyu ${ }^{a}$ Seoung-Su Nah ${ }^{b}$
}

Divisions of a Pulmonology and ${ }^{b}$ Rheumatology, Department of Internal Medicine, Soonchunhyang University College of Medicine, Cheonan, South Korea

\section{Established Facts}

- In most cases, diffuse alveolar hemorrhage (DAH) develops in established systemic lupus erythematosus (SLE) patients with other active organ involvement, which often accompanies the risk of infection.

- Treatment of DAH is demanding because of the high early mortality rate even with aggressive immunosuppressive therapies.

\section{Novel Insights}

- We describe the case of an SLE patient whose cardinal manifestation was DAH which dramatically improved with rituximab without cyclophosphamide or other immunosuppressive agents.

\section{Key Words}

Systemic lupus erythematosus · Diffuse alveolar hemorrhage $\cdot$ Rituximab

\begin{abstract}
Diffuse alveolar hemorrhage (DAH) is a rare but devastating complication of systemic lupus erythematosus (SLE) with a high early mortality rate. DAH seldom occurs without active organ involvement. Recently, rituximab (RTX), a B cell-targeted therapy, has been reported to be effective for life-threatening autoimmune diseases. We describe a SLE patient
\end{abstract}

who presented with acute respiratory failure due to DAH without other active organ involvement. This condition was dramatically improved with RTX without cyclophosphamide.

(c) 2014 S. Karger AG, Basel

\section{KARGER 125}

(c) 2014 S. Karger AG, Basel

0025-7931/14/0891-0062\$39.50/0

E-Mail karger@karger.com

www.karger.com/res

\section{Introduction}

Diffuse alveolar hemorrhage (DAH) occurs in only $5.4 \%$ of systemic lupus erythematosus (SLE) patients, although its mortality rate reaches $61.9 \%$ [1]. In response, 
Fig. 1. Chest X-ray (PA view) on admission showing diffuse alveolar infiltrates (a) and 1 week after RTX treatment (b).
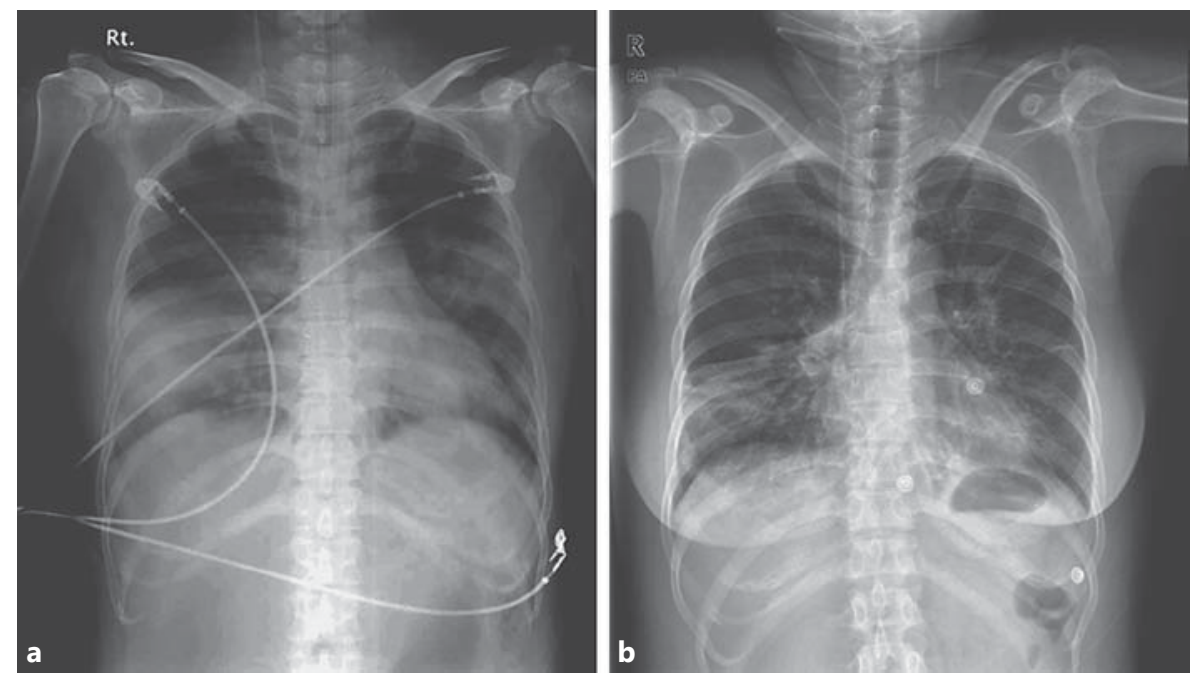

aggressive immunosuppressive therapies such as cyclophosphamide are commonly used. However, the treatment of DAH is demanding with no established treatment regimen, and it occurs in the setting of active other organ involvement and with the risk for serious infection. Rituximab (RTX), which is a specific anti-CD20 antigen $B$ cell antibody, has recently been used as a rescue therapy in life-threatening complications or reparatory autoimmune diseases [2]. Here we present the case of a patient with severe DAH in SLE who did not show any response to steroid treatment and was successfully treated with RTX.

\section{Case Report}

A 37-year-old female presented at our emergency room with severe resting dyspnea and high fever above $40.2^{\circ} \mathrm{C}$ which had developed abruptly within $5 \mathrm{~h}$. She complained of photosensitivity and arthritis in both shoulder joints. She had experienced weight loss of $5 \mathrm{~kg}$ over the past 2 months. An initial arterial blood gas analysis showed severe hypoxemia $\left(\mathrm{PaO}_{2}=56.6 \mathrm{~mm}\right.$ $\mathrm{Hg}$ ), and the chest X-ray showed new diffuse bilateral pulmonary infiltrates (fig. 1). She underwent intubation and mechanical ventilation was initiated. Abundant bloody secretion drained through the endotracheal tube, and her hemoglobin dropped from 9.8 to $7.9 \mathrm{~g} / \mathrm{dl}$ within $24 \mathrm{~h}$. The laboratory examination showed marked leukocytosis $(18,590 / \mu \mathrm{l})$ and thrombocytopenia $(46,000 / \mu \mathrm{l})$ with increased inflammatory serum markers, but her liver function test, serum creatinine levels and urine analysis were normal. An antinuclear antibodies titer was above 1:640 and showed a speckled pattern with positive anti-dsDNA antibody $(>1: 160)$, antiSmith antibody, anti SS-A/Ro and anti SS-B/La, while rheumatoid factor, anti-phospholipid antibodies, anti-GBM antibody and ANCA were all negative. Streptococcal urinary antigen was positive and blood culture revealed Gram-positive cocci, which proved to be Streptococcus pneumoniae. As pulmonary hemorrhage rarely occurs in pneumococcal pneumonia, she was diagnosed as DAH associated with SLE, which might have been triggered by infection.

Empirical antibiotics with $1 \mathrm{~g}$ /day intravenous methylprednisolone pulse were promptly commenced. However, despite this treatment, her condition deteriorated and her oxygen saturation was barely maintained above $90 \%$ even with $100 \%$ oxygen inhalation. The chest X-rays showed aggravation of the diffuse and bilateral infiltration with further decreased hemoglobin levels and platelet count. RTX $500 \mathrm{mg}\left(375 \mathrm{mg} / \mathrm{m}^{2}\right)$ was administrated intravenously on days 3 and 10, in addition to empirical antibiotics and steroid therapy. The day after the first RTX injection, the patient's clinical symptoms, including hemoptysis and oxygen demand, were improved. Glucocorticoid pulse therapy (methylprednisolone $1 \mathrm{~g} /$ day) was continued for a total of 5 days and was tapered to methylprednisolone $55 \mathrm{mg} /$ day. On day 6, 3 days after the first RTX injection, mechanical ventilation was discontinued and the platelet count normalized. A week later, the infiltration evident on chest X-ray was markedly reduced (fig. 1). She was subsequently discharged on prednisolone $55 \mathrm{mg} /$ day which has been gradually reduced to $5 \mathrm{mg} /$ day over 6 months. To date, no further SLE flares including DAH have occurred after 4 years of surveillance.

\section{Discussion}

Pulmonary disease is a common manifestation of SLE, which over half of patients may experience during the course of the disease [3]. The most common pulmonary manifestation is pleuritis, but the most devastating pulmonary complication is pulmonary hemorrhage with a mortality rate reported to be as high as $70-90 \%$ $[4,5]$. In most cases DAH develops in patients with 
Table 1. Case summaries of RTX treatment for DAH in patients with SLE

\begin{tabular}{|c|c|c|c|c|c|c|}
\hline $\begin{array}{l}\text { Age, } \\
\text { years }\end{array}$ & Sex & $\begin{array}{l}\text { Disease } \\
\text { duration } \\
\text { (SLE), years }\end{array}$ & Concurrent treatment for DAH & RTX timing and dosing & Outcome & $\begin{array}{l}\text { Reference } \\
\text { No. }\end{array}$ \\
\hline 29 & $\mathrm{~F}$ & 12 & GC, CYC, plasma exchange & every 2 weeks for 6 weeks, $375 \mathrm{mg} / \mathrm{m}^{2}$ & survived & [9] \\
\hline 18 & M & 6 & GC, MMF & 2 weeks apart, $375 \mathrm{mg} / \mathrm{m}^{2}$ & survived & {$[10]$} \\
\hline 23 & $\mathrm{~F}$ & 5 & GC, CYC, MMF, AZA, plasma exchange & 2 weeks apart, $1 \mathrm{~g}$ & deceased & [11] \\
\hline 37 & $\mathrm{~F}$ & present case & GC & 1 week apart, $375 \mathrm{mg} / \mathrm{m}^{2}$ & survived & \\
\hline
\end{tabular}

$\mathrm{AZA}=$ Azathioprine $\mathrm{CYC}=$ cyclophosphamide $\mathrm{GC}=$ glucocorticoid $\mathrm{MMF}=$ mycophenolate mofetil; N/A = not available .

established SLE, often in the setting of active other organ involvement, including nephritis or neuropsychiatric lupus $[1,4,5]$. In the current case, the patient presented with DAH as the first clinical manifestation of SLE without lupus nephritis or other organ involvement. Although blood and sputum culture revealed pneumococcal infection, considering the fact that DAH is rarely observed in pneumococcal infection, DAH was associated with SLE rather than with pneumococcal infection.

The current regimen for treatment of DAH in SLE includes high-dose steroids with either cyclophosphamide or azathioprine and plasmapheresis, targeting both (B cell and $\mathrm{T}$ cell) branches of the immune system [6]. However, there have been no randomized controlled trials comparing the various treatment modalities of DAH in patients with SLE. RTX is a chimeric monoclonal antibody against CD20 that is expressed in most of the stages of B cells. Considering that an acquired loss of B cell tolerance resulting in dysregulation of antibody production may lead to the many features of SLE, RTX can be a rescue therapy in the treatment of SLE $[2,7,8]$. So far, 6 other cases of SLE-induced DAH have been reported to respond to RTX administration (table 1), where RTX was administered concomitantly with other immunosuppressive therapies such as cyclophosphamide or mycophenolate mofetil [6,9-13]. Although aggressive immunosuppressive therapy is necessary for treating $\mathrm{DAH}$, it is challenging because of the high risk for infection. Indeed, it was reported that more than half of patients with pulmonary hemorrhage and SLE had concurrent infection with a genus of bacteria such as Pseudomonas, Serratia, Stenotrophomonas and Aspergillus [14]. In the present case, due to the coexis- tence of pneumococcal infection, RTX without other immunosuppressive therapies was administered, which resulted in a very rapid response to RTX treatment. The mechanism of early improvement by RTX has not been elucidated, but, since circulating B cell depletion normally occurs within $48 \mathrm{~h}$ of RTX administration [8], it is supposed to be associated with the suppression of cytokine production and inhibition of T-B cell cooperation [15]. To our knowledge, this is the first case of the successful treatment of DAH in SLE with RTX monotherapy.

In summary, DAH can be the first clinical manifestation of SLE and RTX should be considered as a treatment option in the case of concurrent infection, limiting the use of other immunosuppressants.

\section{Financial Disclosure and Conflicts of Interest}

This work was supported by Soonchunhyang University Research Fund.

\section{References}

\footnotetext{
1 Kwok SK, Moon SJ, Ju JH, et al: Diffuse alveolar hemorrhage in systemic lupus erythematosus: risk factors and clinical outcome: results from affiliated hospitals of Catholic University of Korea. Lupus 2011;20:102-107.

2 Braun-Moscovici Y, Butbul-Aviel Y, Gualnik L, et al: Rituximab: rescue therapy in lifethreatening complications or refractory autoimmune diseases: a single center experience. Rheumatol Int 2013;33:1495-1504.

-3 Pego-Reigosa JM, Medeiros DA, Isenberg DA: Respiratory manifestations of systemic lupus erythematosus: old and new concepts. Best Pract Res Clin Rheumatol 2009;23:469480.
} 
4 Badsha H, Teh CL, Kong KO, Lian TY, Chng HH: Pulmonary hemorrhage in systemic lupus erythematosus. Semin Arthritis Rheum 2004;33:414-421.

5 Kamen DL, Strange C: Pulmonary manifestations of systemic lupus erythematosus. Clin Chest Med 2010;31:479-488.

-6 Narshi CB, Haider S, Ford CM, Isenberg DA, Giles IP: Rituximab as early therapy for pulmonary haemorrhage in systemic lupus erythematosus. Rheumatology (Oxford) 2009; 49:392-394.

7 Smith KG, Jones RB, Burns SM, Jayne DR: Long-term comparison of rituximab treatment for refractory systemic lupus erythematosus and vasculitis: remission, relapse, and re-treatment. Arthritis Rheum 2006;54:29702982.
-8 Lee JW, Kim HA, Sung JM, Suh CH: Successful treatment of refractory immune thrombocytopenia with anti-CD20 antibody in a patient with systemic lupus erythematosus. Lupus 2009; 19:227-228.

9 Nellessen CM, Pöge U, Brensing KA, Sauerbruch T, Klehr HU, Rabe C: Diffuse alveolar haemorrhage in a systemic lupus erythematosus patient successfully treated with rituximab: a case report. Nephrol Dial Transplant 2008;23:385-386.

10 Pottier V, Pierrot M, Subra JF, et al: Successful rituximab therapy in a lupus patient with diffuse alveolar haemorrhage. Lupus 2011;20: 656-659.

11 Todd DJ, Costenbader KH: Dyspnoea in a young woman with active systemic lupus erythematosus. Lupus 2009;18:777-784.
2 Martinez-Martinez MU, Abud-Mendoza C: Recurrent diffuse alveolar haemorrhage in a patient with systemic lupus erythematosus: long-term benefit of rituximab. Lupus 2012; 21:1124-1127.

13 Pinto LF, Candia L, Gagcia P, et al: Effective treatment of refractory pulmonary hemorrhage with monoclonal anti-CD20 antibody (rituximab). Respiration 2009;78: 106-109.

14 Rojas-Serrano J, Pedroza J, Regalado J, et al: High prevalence of infections in patients with systemic lupus erythematosus and pulmonary haemorrhage. Lupus 2008;17:295299.

15 McFarland HF: The B cell - old player, new position on the team. N Engl J Med 2008;358: 664-665. 